\title{
TWO COUNTEREXAMPLES INVOLVING INNER FUNCTIONS
}

\author{
KENNETH STEPHENSON
}

\begin{abstract}
Two questions about the existence of bounded analytic functions with prescribed behavior are answered using a construction technique of McLaughlin and Piranian. If $A$ is a relatively closed, countable subset of the unit disc, the construction gives

(I) Function $f \in H^{\infty}$ so that the inner factor of $f-\alpha$ is a finite Blaschke product if and only if $\alpha \in A$.

(II) Inner function $\phi$ so that $\phi-\alpha$ has a discrete singular inner factor if and only if $\alpha \in A$.
\end{abstract}

A technique of McLaughlin and Piranian [2] is used to construct bounded analytic functions in the unit disc $\mathscr{U}$ whose inner factors have prescribed properties. We refer to [3] for definitions of the various types of inner functions. If $f \in H^{\infty}(\mathcal{U})$, then $\operatorname{Inn}(f)$ is used to denote the inner factor of $f$.

Let $A$ be a relatively closed and countable subset of $थ$. For convenience and without loss of generality, we assume $0 \in A$.

EXAMPLE I. There exists a function $f \in H^{\infty}(\mathcal{U}), f(\mathcal{U})=\mathscr{U}$, so that $\operatorname{Inn}(f-\alpha)$ is a nonconstant finite Blaschke product if and only if $\alpha \in A$.

EXAMPLE II. There exists a discrete singular inner function $\phi$ so that $\operatorname{Inn}(\phi-\alpha)$ has a discrete singular inner factor if and only if $\alpha \in A$.

Example I shows that a result of Carl Cowen [1, Corollary to Theorem 5] on commutants of analytic Toeplitz operators is strictly stronger than the earlier result of Thomson [4]. Specifically, if $A=\{0\}$, then the set

$$
\{\beta \in \mathscr{Q}: \operatorname{Inn}(f-f(\beta)) \text { is finite Blaschke }\}
$$

is the finite set $f^{-1}(\{0\})$.

Example II answers negatively the final question in [3]. For $\alpha \in \mathcal{Q}$, let $\psi_{\alpha}$ be the Möbius transformation of $\mathcal{Q}$ given by

$$
\psi_{\alpha}(z)=(z-\alpha) /(1-\bar{\alpha} z), \quad z \in \mathcal{U} .
$$

Let $A=\{0\} \cup\{1 / n: n=2,3, \ldots\}$. Then the inner function $\phi$ given by Example II is the limit in $H^{\infty}$ of the sequence $\left\{\left(\psi_{1 / n} \circ \phi\right): n=2,3, \ldots\right\}$, and each of these has a nontrivial discrete singular inner factor.

Construction of Example I. Fix $\beta \in \mathcal{U}$ so that the line segment $\Gamma=[\beta, 1)$ is contained in $\mathscr{Q} \backslash A$. Choose a sequence $\left\{\Omega_{k}: k=0,1, \ldots\right\}$ of open

Received by the editors March 13, 1978.

AMS (MOS) subject classifications (1970). Primary 30A76.

Key words and phrases. Bounded analytic function, inner function, Riemann surface. 
subsets of $\mathscr{U}$ so that for $k=0,1,2, \ldots$ we have

(a) $\Gamma \subseteq \Omega_{k} \subseteq \mathcal{Q} \backslash A$,

(b) $\bar{\Omega}_{k} \cap \mathscr{Q} \subseteq \Omega_{k+1}$,

(c) $\Omega_{k}$ is connected, with $\Omega_{0}$ not simply connected, and

(d) $\cup_{k=0}^{\infty} \Omega_{k}=\mathscr{U} \backslash A$.

Denote by $S_{k}$ the universal covering surface of $\Omega_{k}, k=0,1,2, \ldots$ Let $\gamma_{0}, \gamma_{1}, \ldots$ be a labelling of the segments on $S_{0}$ lying over $\Gamma$. For each $k=1,2, \ldots$, slit one sheet of $S_{k}$ along a segment lying over $\Gamma$, slit $S_{0}$ along $\gamma_{k}$, and join $S_{k}$ to $S_{0}$ by connecting opposite sides of these slits. Likewise, slit a copy of $\mathcal{Q}$ along $\Gamma$ and join it to $S_{0}$ along $\gamma_{0}$. Let $S$ denote the resulting simply connected Riemann surface with $\pi$ the projection onto $\mathcal{Q}$. Let $F$ be a conformal mapping of the unit disc onto $S$. We show that $f=\pi \circ F$ has the desired properties.

Clearly $f \in H^{\infty}$ and $f(\mathcal{Q})=\mathscr{Q}$. If $\alpha \in \mathscr{Q} \backslash A$, then there is some $k_{0}$ with $\alpha \in \Omega_{k_{0}}$; hence $\alpha \in \Omega_{k}$ for all $k>k_{0}$, implying that $f-\alpha$ has infinitely many zeros. On the other hand, if $\alpha \in A$, then $f-\alpha$ has precisely one zero, and it remains only to show that $f-\alpha$ has no (nontrivial) singular inner factor.

Removing the sets $F^{-1}\left(\gamma_{k}\right), k=0,1,2, \ldots$ breaks $\mathscr{Q}$ into disjoint simply connected open sets $\Delta_{0}, \Delta_{1}, \ldots$, each $\Delta_{k}$ corresponding under $F$ to the slit surface $S_{k}$, and $\Delta_{-1}$, corresponding under $F$ to the slit copy of $\mathscr{U}$ which went into $S$. Suppose there exists $e^{i \theta} \in \partial \mathscr{Q}$ and $r_{0}, 0<r_{0}<1$, so that

$$
\left|f\left(r e^{i \theta}\right)-\alpha\right|<\delta, \quad r_{0} \leqslant r<1,
$$

where $\delta=\delta(\alpha)>0$ is given by

$$
\delta=\inf \{|\alpha-z|: z \in \Gamma \cup \partial \mathscr{Q}\} .
$$

Then the segment $\left\{r e^{i \theta}: r \geqslant r_{0}\right\}$ lies entirely within one of $\Delta_{-1}, \Delta_{0}, \Delta_{1}, \ldots$ It cannot lie in $\Delta_{-1}$ since the image $\left\{F\left(r e^{i \theta}\right): r \geqslant r_{0}\right\}$ would then be contained in a compact subset of $S$, contradicting the fact that $F$ is a conformal mapping. Thus it lies in $\Delta_{k_{0}}$ for some $k_{0} \geqslant 0$. But this implies that $\left\{f\left(r e^{i \theta}\right)\right.$ : $\left.r \geqslant r_{0}\right\} \subseteq \Omega_{k_{0}}$. The conditions imposed on the sets $\left\{\Omega_{k}\right\}$ imply $\alpha \notin \Omega_{k_{0}}$, so

$$
\liminf _{r \rightarrow 1}\left|f\left(r e^{i \theta}\right)-\alpha\right|>0 \text {. }
$$

Since (1) implies (2) we conclude that $f-\alpha$ cannot have zero as a radial limit. As is well known, this implies $f-\alpha$ has no singular inner factor.

Construction of Example II. Fix a segment $\Gamma$ as above. Let $\alpha_{1}, \alpha_{2}, \ldots$ be the nonzero points of $A$. (Of course, $A$ may be finite, but that just makes our proof easier.) Let $S_{0}$ be the universal covering surface of $\mathscr{U} \backslash\{0\}$ and $S_{k}$ the universal covering surface of $\mathscr{U} \backslash\left\{0, \alpha_{k}\right\}, k=1,2, \ldots$ Let $\gamma_{1}, \gamma_{2}, \ldots$ denote the segments on $S_{0}$ lying over $\Gamma$. As before, slit one sheet of each $S_{k}$ along a segment lying over $\Gamma$, slit $S_{0}$ along $\gamma_{k}$, and join $S_{k}$ to $S_{0}$ by connecting opposite sides of these slits. Let $S$ denote the resulting simply connected Riemann surface, $\pi$ the projection onto $\mathscr{Q} \backslash\{0\}$, and $G$ a conformal 
mapping of the unit disc onto $S$. We show that $\phi=\pi \circ G$ has the desired properties.

First, all radial limits of $\phi$ lie in $A \cup \partial \mathcal{Q}$. $A$ is countable, hence of capacity zero; so the set $\left\{e^{i \theta}: \lim _{r \rightarrow 1} \phi\left(r e^{i \theta}\right) \in A\right\}$ is of Lebesgue measure zero. That is, almost all radial limits of $\phi$ are in $\partial \mathcal{Q}$, so $\phi$ is an inner function. Also, if $\alpha \in \mathcal{U} \backslash A$, then $\alpha$ cannot be a radial limit of $\phi$, implying that zero cannot be a radial limit of $\phi-\alpha$. Thus $\phi-\alpha$ has no singular inner factor. $\phi$ itself is singular since $S$ does not cover $z=0$.

Now fix $\alpha_{k} \in A$. As before, removing the sets $G^{-1}\left(\gamma_{k}\right)$ breaks $\mathcal{U}$ into disjoint simply connected open sets $\Delta_{0}, \Delta_{1}, \ldots$, each $\Delta_{k}$ corresponding under $G$ to the slit surface $S_{k}$. $\alpha_{k}$ occurs as the asymptotic value on some arc in $\mathscr{Q}$. An argument similar to that used earlier shows that from some point on, such an arc lies entirely in $\Delta_{k}$. Also, since $\alpha_{k}$ is a positive distance from $\Gamma$, such an arc must end at an interior point $e^{i \theta} \in \partial \Delta_{k} \cap \partial \mathcal{Q}$. We see then that $\phi-\alpha$ does not vanish in $\Delta_{k}$, yet $\lim _{r \rightarrow 1}\left(\phi\left(r e^{i \theta}\right)-\alpha\right)=0$; so $\phi-\alpha$ has a singular inner factor. The counting argument used in [3] to prove Theorem I(a) can be applied here to show that the number of such points $e^{i \theta}$ in the interior of $\partial \Delta_{k} \cap \partial \mathscr{U}$ is at most countable. Since these are the support points for the measure associated with the singular factor of $\phi$, that factor must be a discrete singular inner function.

Finally, the same counting argument shows that $\left\{e^{i \theta}: \lim _{r \rightarrow 1} \phi\left(r e^{i \theta}\right)=0\right\}$ is at most countable, hence $\phi$ itself is a discrete singular inner function.

\section{REFERENCES}

1. Carl. C. Cowen, The commutant of an analytic Toeplitz operator, Trans. Amer. Math. Soc. 239 (1978), 1-31.

2. Renate McLaughlin and George Piranian, The exceptional set of an inner function, Sitzungsber. Österreich. Akad. Wiss. Math.-Natur. Kl., Abteilung II, Band 185, 1976.

3. Kenneth Stephenson, Omitted values of singular inner functions, Michigan Math. J. 25 (1978), 91-100.

4. James E. Thomson, The commutant of a class of analytic Toeplitz operators. II, Indiana Univ. Math. J. 25 (1976), 793-800.

Department of Mathematics, University of Tennessee, Knoxville, Tennessee 37916 